\pm PCI was attempted. PCI was done in 36 (95\%). 14 (37\%) were in cardiogenic shock, 9 (24\%) were transferred to ITU, 5 (13.2\%) had therapeutic cooling and $33(87 \%)$ survived to hospital discharge. In $20 \mathrm{pts}$, the diagnosis of STEMI was made following resuscitation (Gp 2). Of these 18 (90\%) had angiography + PCI, 9 (45\%) were in cardiogenic shock, $6(30 \%)$ had therapeutic cooling, $12(60 \%)$ admitted to ITU, 3 (15\%) were admitted to ITU first before coming to the Cath lab and 15 (75\%) survived to discharge. There was no evidence of STEMI following resuscitation in 18 pts accepted by our unit (Gp 3). Of these, 17 (94\%) had angiography, 7 (39\%) had PCI, 2 $(18 \%)$ had CABG, 2 (18\%) were in cardiogenic shock, 15 (83\%) were admitted to ITU, 7 (38\%) had therapeutic cooling and survival to discharge was $94 \%$. In Gp 3, angiography was delayed pending a head CT in 7 (38\%) compared to none in Gps 1 and 2. Angiography was deferred following initial ITU treatment in $8(44 \%)$ patients in Gp 3. Overall survival in patients with and without cardiogenic shock was $58 \%$ and $98 \%$ respectively $(p<0.01)$. Survival was $87 \%$ for those with a witnessed OOHA compared to $40 \%$ where the arrest was not witnessed $(p<0.05)$.

Conclusion A programme of immediate cardiovascular assessment of patients with $\mathrm{OOHA}$ and referral for angiography and revascularisation as deemed appropriate is associated with encouraging shortterm outcomes. Outcomes are related to the presence of shock and whether the OOHA was witnessed or not. National guidance on the immediate management of these patients may improve outcomes.

\section{MANAGEMENT OF BIFURCATION DISEASE WITH A DEDICATED SIDE-BRANCH DEVICE; A SINGLE CENTRE EXPERIENCE WITH THE TRYTON STENT}

doi:10.1136/heartjnl-2012-301877b.30

V Venugopal, * R Singh, J Martins, M Norell. Royal Wolverhampton Hospital NHS Trust, UK

Background The optimal strategy for the treatment of bifurcation disease is not yet defined. While trial data suggests that "provisional" side-branch (SB) stenting may be superior to more complex 2-stent approaches, the role of customised devices designed specifically to treat this complex anatomy has not been examined. The TRYTON side-branch stent is one such example. This is a 5 French guide compatible, non-drug eluting, Cobalt Chromium device mounted on a monorail balloon catheter. When deployed into the SB, it is specifically designed to scaffold the ostium. A second stent is mandated in the main branch $(\mathrm{MB})$ and deployed through TRYTON in a culottes fashion, serves to further cover the carina and secure TRYTON. This is followed by final kissing balloon inflation.

Methods All patients undergoing bifurcation stenting using the TRYTON stent were systematically included in a registry. Followup data were obtained by case note and angio review and telephone interview.

Results Since November 2008, 79 patients (mean age 63 years, range 36-84) with bifurcation disease and SB vessel diameters of at least $2.5 \mathrm{~mm}$ were selected for treatment with TRYTON in our centre. $75 \%(59 / 79)$ of the target lesions were in the left anterior descending/diagonal while 4 were in the left main stem. $60 \%$ (47/ 79) lesions were Medina 1.1.1, while 15\% (12/79) had no SB disease. Procedural success was achieved in $94 \%$. In 5 cases from our initial 6 months' experience, TRYTON could not be delivered to the target lesion due to proximal calcification or tortuosity, resulting in stent dislodgement in 4 cases and requiring retrieval of TRYTON in 1. An alternate bifurcation strategy was used and none of the 5 patients suffered clinical sequelae. Subsequent device modification by the manufacturers to improve balloon adherence, trackability and deliverability resulted in a $100 \%$ success rate. In the remaining 74 patients TRYTON was deployed successfully and in all but one case, a drug eluting stent was used in the main branch. Additional drug eluting stents were deployed into further diseased segments of the $\mathrm{SB}$ in five cases and the $\mathrm{MB}$ in 28. Final kissing balloon inflation could be performed in all but one case (99\%). Angiographic success in both the main and side branches was $100 \%$. After a median follow-up of 18 months (range 2-40), there have been no instances of unplanned readmission with ischaemia, myocardial infarction or death in any of the 79 patients. In one case in-stent restenosis in the bare-metal $\mathrm{MB}$ stent required further treatment with a drug eluting balloon.

Conclusion TRYTON performs predictably and successfully scaffolds the side-branch ostium. The enhanced ability to rewire the side-branch for final kissing balloon inflation when compared with other 2-stent strategies may have contributed to our low observed clinical event rate. The medium term results in this series are encouraging. A randomised trial comparing TRYTON with provisional side-branch stenting is ongoing.

\section{USE OF THE NOVEL SIDEGUARD DEDICATED BIFURCATION STENT: A REAL WORLD EXPERIENCE}

doi:10.1136/heartjnl-2012-301877b.31

S Sastry, ${ }^{*}$ M Mamas, K Palmer, H lles-Smith, M El-Omar, D Fraser, F Fath-Ordoubadi. Manchester Royal Infirmary, Manchester, UK

Introduction PCI treatment of bifurcation disease is technically challenging. Dedicated bifurcation stents have been developed to address some of the challenges associated with bifurcation lesions. The aim of this study is to report clinical utility and outcomes of the Sideguard stent in patients undergoing treatment to bifurcation coronary lesions in a real world setting in a large tertiary UK centre. Methods Retrospective study of 61 consecutive patients treated with the Sideguard Stent for bifurcation PCI at the Manchester Heart Centre from March 2010 to October 2011. Patient demographics and PCI procedural data were obtained from the in-house, cross validated PCI database. Mortality data were obtained from National Office of Statistics. Interim 6 month and 1 year data were obtained from clinical review. Clinical end point studied included death, subsequent myocardial infarction, stent thrombosis and target lesion/vessel revascularisation (TLR/TVR).

Results A total of 61 consecutive patients underwent bifurcation PCI using the Sideguard stent during this study. Mean age $57.1 \pm 11.5$ years (range $30-76$ ) of which $50 / 61$ were male $(81.9 \%)$. Bifurcation site was in LAD in 44 (72.2\%), Cx in $14(22.9 \%)$ and RCA in $3(4.9 \%)$ of cases. Medina classification was 1:1:1 in 38 (62.3\%), 1:0:1 in $10(16.3 \%)$ and 0:1:1 in $6(9.9 \%)$ of cases. Moderate or severe calcification was present in $27(44.3 \%)$ of cases. Mean main vessel (MV) diameter was $3.36 \pm 0.39 \mathrm{~mm}$, mean $\mathrm{MV}$ stent length was $35 \pm 18.4 \mathrm{~mm}$ and mean number of stents in $M V$ was 1.54 \pm 0.77 . Mean Side branch vessel (SB) diameter was $2.93 \pm 0.33 \mathrm{~mm}$, mean SB stent length was $13.4 \pm 8.5 \mathrm{~mm}$ and mean number of stents in SB was $1.24 \pm 0.43$. Final kissing balloon was attempted in 57 (93.4\%) and successful in all cases attempted. Procedural complications occurred in 4/61 (6.6\%) cases. Complications included 1 case of limited perforation of the $M B$ which was treated conservatively, 1 case of clot formation related to IVUS examination (neither of these complications were thought to be related to sidegurad stent, directly) and 2 cases of stent displacement. Local deployment techniques have been modified to combat displacement. Operators now wait for $30 \mathrm{~s}$ and take a frame shot after stent deployment to confirm placement before removing the stent balloon. In our interim analysis 43 of these 61 patients have been followed up for at least 6 months $(70.5 \%)$ and 37 patients for at least 1 year $(60.7 \%)$. There were no deaths or stent thromboses. 